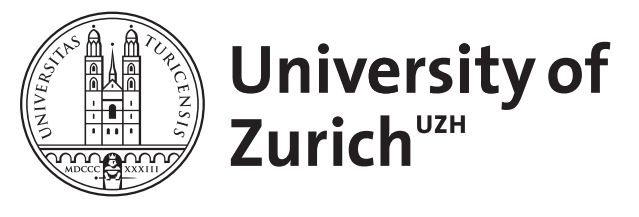

\title{
Die infektiöse Spondylitis
}

Huttner, B ; Opravil, M

\begin{abstract}
Bei der infektiösen Spondylitis handelt es sich in der Regel um eine Osteomyelitis von 2benachbarten Wirbelkörpern unter Beteiligung der Zwischenwirbelscheibe (Spondylodiszitis). Am häufigsten ist die Lendenwirbelsäule betroffen, gefolgt von der Brustwirbelsäule. Die Symptome der infektiösen Spondylitis sind unspezifisch, was nicht selten zu einer Verzögerung von einigen Wochen bis zur Diagnosestellung führt. Die infektiöse Spondylitis kann durch eine Vielzahl von Erregern bedingt sein, die überwiegend auf hämatogenem Weg die Wirbelsäule erreichen. Der häufigste Erreger ist Staphyloccocus aureus. Die Spondylitis ist zudem die häufigste skelettale Manifestation der Tuberkulose. Wie bei anderen Formen der Osteomyelitis ist die Kenntnis des Erregers für die Wahl einer adäquaten Therapie entscheidend, weshalb eine mikrobiologische Diagnose auf jeden Fall angestrebt werden sollte. Die Mehrheit der Fälle kann konservativ mit antibiotischer Therapie geheilt werden
\end{abstract}

DOI: https://doi.org/10.1007/s00393-005-0017-0

Posted at the Zurich Open Repository and Archive, University of Zurich

ZORA URL: https://doi.org/10.5167/uzh-155902

Journal Article

Published Version

Originally published at:

Huttner, B; Opravil, M (2006). Die infektiöse Spondylitis. Zeitschrift für Rheumatologie, 65(1):7-11.

DOI: https://doi.org/10.1007/s00393-005-0017-0 
Z Rheumatol 2006 65:7-11 DOI 10.1007/s00393-005-0017-0

Online publiziert: 19. Januar 2006

(c) Springer Medizin Verlag 2006

\author{
B. Huttner ${ }^{1,2} \cdot$ M. Opravil ${ }^{1}$ \\ ${ }^{1}$ Klinik für Infektionskrankheiten und Spitalhygiene, Departement für Innere Medizin, \\ Universitätsspital Zürich \\ ${ }^{2}$ Klinik für Infektionskrankheiten und Spitalhygiene, Departement für Innere Medizin, \\ Universitätsspital Zürich
}

\title{
Die infektiöse Spondylitis
}

\section{Pathogenese}

In den meisten Fällen entsteht die Infektion der Wirbelkörper auf hämatogenem Weg. Als primäre Infektionsquelle sind vor allem Haut- und Weichteilinfekte, Harnwegsinfekte, Endokarditiden, Infektionen der Atemwege und katheterassoziierte Infektionen beschrieben. Nicht in allen Fällen kann jedoch eine Infektionsquelle ausfindig gemacht werden [3].

Aufgrund der besonderen Anatomie der Gefäßversorgung sind meist 2 benachbarte Wirbelkörper betroffen. In der Folge ist auch die Zwischenwirbelscheibe in den infektiösen Prozess einbezogen (Spondylodiszistis). Bei Kindern und bei degenerativen Veränderungen der Bandscheiben sind diese vaskularisiert [11], was einen direkten Befall möglich macht.

Die häufigste Lokalisation ist die Lendenwirbelsäule, gefolgt von der Brust- und Halswirbelsäule. Die tuberkulöse Spondylitis zeigt weniger eine Prädilektion für die Lendenwirbelsäule, sodass bei Befall der Brust- und Halswirbelkörper auch an eine tuberkulöse Genese gedacht werden sollte [2].

Eine infektiöse Spondylitis kann zudem nach operativen Eingriffen an der Wirbelsäule als Komplikation auftreten, deren Behandlung sich aufgrund des häufig anwesenden Fremdmaterials schwierig gestalten kann. Um so wichtiger ist eine adäquate perioperative Antibiotikaprophylaxe, die das Infektionsrisiko erwiesenermaßen senkt [12].

\section{Mikrobiologie}

Es gibt fast keine Bakterien oder Pilze, die noch nicht als Erreger einer vertebralen Osteomyelitis beschrieben wurden $(\boldsymbol{\bullet}$ Tabelle 1). Wie bei Osteomyelitiden der anderen Knochen sind Staphylokokken (vor allem Staphylococcus aureus, aber auch Koagulase-negative Staphylokokken) zumindest in den industrialisierten Ländern die am häufigsten isolierten Keime. Bei bestimmten Risikogruppen, wie drogenabhängigen und immunsupprimierten Personen, sowie nach operativen Eingriffen am Achsenskelett müssen auch Gram-negative Bakterien (z. B. Escherichia coli) und Pilze (vor allem Candida spp.) berücksichtigt werden [10]. Bei Patienten mit Diabetes mellitus werden vermehrt betahämolysierende Streptokokken als Erreger identifiziert [13].

In den ärmeren Weltregionen ist die Spondylitis durch Mycobacterium tuberculosis immer noch verbreitet. Ebenso ist bei entsprechender Exposition (Kontakt mit Tieren oder Tierprodukten) und passender geographischer Anamnese an die Brucellose zu denken, bei der die Spondylitis eine häufige Komplikation darstellt.

\section{Klinik}

Die Patienten stellen sich meist mit lokalisierten Schmerzen im Bereich der Wirbelsäule vor. Der Beginn ist überwiegend subakut mit über einige Wochen progredienten Schmerzen. Typischerweise sind die Schmerzen intial belastungsabhängig und treten auch nachts auf. Insgesamt ist munsuppressiver Therapien mit einer $\mathrm{Zu}$ nahme der Inzidenz zu rechnen. 
Tabelle 1

Häufige Erreger der vertebralen Osteomyelitis

\section{Grampositive Bakterien}

Staphylococcus aureus

Koagulase-negative Staphylokokken

Betahämolysierende Streptokokken

Enterokokken

\section{Gramnegative Bakterien}

Escherichia coli

Nosokomial

Pseudomonas aeruginosa

Brucellen

Anamnese

Entwicklungsländer

Salmonellen

Klebsiella pneumoniae

Proteus mirabilis

Anaerobier

Selten, vor allem postoperativ

Propionibacterium acnes

Bacteroides

Fusobacterium

Mykobakterien

Mycobacterium tuberculosis

Atypische Mykobakterien

Pilze

Weltweit häufig

Immunsuppression

Immunsuppression, postoperativ

Candida

Aspergillen

Kryptokokken
Häufigster Erreger

Häufig postoperativ, vor allem bei Fremdmaterial

Diabetes mellitus als Risikofaktor

Selten die Klinik jedoch wenig spezifisch und die Differenzialdiagnose bei Rückenschmerzen breit. Systemische Symptome, wie erhöhte Temperatur, $\mathrm{Ge}$ wichtsverlust und Nachtschweiß sind eher die Ausnahme. So ist Fieber, ein bekanntes „Alarmsignal“ bei Rückenschmerzen, in weniger als der Hälfte der Fälle vorhanden [16].

Laborchemische Untersuchungen sind zur Diagnosestellung wenig hilfreich, da oft keine oder nur eine geringe Erhöhung der systemischen Entzündungsparameter (CRP, Leukozyten) vorliegt. Die Blutsenkungsgeschwindigkeit ist in der Regel erhöht, jedoch ist deren Aussagekraft durch ihre geringe Spezifität begrenzt. Neurologische Ausfallserscheinungen bei Mitbeteiligung der Nervenwurzeln treten erst im fortgeschrittenen Krankheitsstadium auf. Paravertebrale und epidurale Abszesse sind gefürchtete Komplikationen und treten am häufigsten bei Beteiligung der Halswirbelsäule auf [6].
Zusammenfassend lässt sich sagen, dass die unspezifische Präsentation der infektiösen Spondylitis die klinische Diagnosestellung erschwert. Der betreuende Arzt ist herausgefordert bei entsprechendem Kontext - trotz der relativen Seltenheit dieses Krankheitsbildes - an das Vorliegen einer vertebralen Osteomyelitis zu denken und weitergehende Abklärungen einzuleiten.

\section{Bildgebung}

Das konventionelle Röntgenbild der Wirbelsäule stellt eine relativ kostengünstige und einfache, allerdings wenig sensitive initiale Untersuchung bei Verdacht auf infektiöse Spondylitis dar. Radiologische Zeichen wie die Verschmälerung der Zwischenwirbelräume und Destruktion der Wirbelkörperendplatten lassen sich frühestens nach 2-3 Wochen feststellen und auch dann bei weitem nicht bei allen Fällen [14]. Selten kann ein einzel- ner Wirbelkörper betroffen sein und radiologisch das Bild einer vertebralen Kompressionsfraktur zeigen [9]. Bei entsprechendem klinischem Verdacht sollte ein unauffälliges konventionelles Röntgenbild nicht von weitergehenden Untersuchungen abhalten.

Die radiologische Methode der Wahl zur Diagnose der infektiösen Spondylitis stellt heute die Magnetresonanztomographie (MRT) dar (• Abb. 1), mit der bereits wenige Tage nach Beginn der Symptomatik erste Hinweise gefunden werden können. Zudem erlaubt die MRT neben den knöchernen Strukturen auch die Zwischenwirbelscheibe und die angrenzenden Weichteile mitzubeurteilen, was besonders bei der Diagnose von Komplikationen wie paravertebralen und epiduralen Abszessen wichtig ist. Eine Unterscheidung zwischen tuberkulöser und nichttuberkulöser Osteomyelitis ist jedoch nicht möglich [1].

Die Computertomographie (CT) ist weniger sensitiv, vor allem im Hinblick auf die die Beurteilung von Weichteilveränderungen. Sie kann aber im Gegensatz zur MRT auch bei Anwesenheit von Metallimplantaten durchgeführt werden. In Zukunft dürfte die Positronenemissionstomographie (PET) nach Gabe von ${ }^{18} \mathrm{~F}$ Fluorodeoxyglukose (FDG-PET) in Kombination mit der CT (PET-CT) an Bedeutung gewinnen. Bei der Unterscheidung degenerativer und infektiöser Prozesse der Zwischenwirbelscheiben zeigt sie sich der MRT überlegen [15], die Erfahrungen hinsichtlich ihrer Sensitivität und Spezifität sind aber noch begrenzt.

Zudem stehen verschiedene szinitigraphische Methoden zur Verfügung, die eine hohe Sensitivität aufweisen, deren Nachteile aber in einer niedrigen Spezifität und geringen räumlichen Auflösung liegen. Manche Autoren empfehlen die Durchführung einer Gallium-Szinitigraphie, falls die MRT nicht konklusiv ist oder aus bestimmten Gründen nicht durchgeführt werden kann [8].

\section{Differenzialdiagnose}

Wie erwähnt umfasst die Differenzialdiagnose der infektiösen Spondylitis ein breites Spekturm degenerativer und neoplastischer Erkrankungen. Die erosive 
Osteochondrose, eine entzündliche Degeneration der Wirbelkörper, betrifft jedoch meist die tiefen lumbalen Abschnitte und zeigt in der MRT ein chrarakteristisches Bild [14].

\section{Mikrobiologische Diagnostik}

Falls sich mittels Blutkulturen keine mikrobiologische Diagnose stellen lässt, sollte eine CT-gesteuerte perkutane Biopsie vorgenommen werden, um Material für Mikrobiologie (aerobe und anaerobe Kulturen, Mykobakterienkulturen, Pilzkulturen) und Histopathologie zu gewinnen. Bei klinischem Verdacht auf seltene bzw. schwer zu kultivierende Erreger wie Brucellen sollte man vorher mit dem Mikrobiologielabor Rücksprache nehmen. Leider kann auf diese Weise nur in etwa der Hälfte der Fälle erfolgreich ein Erreger identifiziert werden [10], sodass manche Autoren bei negativen mikrobiologischen Ergebnissen eine Wiederholung dieser Prozedur empfehlen [8]. Eine offene Biopsie sollte aufgrund der höheren Komplikationsrate für Fälle vorbehalten bleiben, die auf die empirische Therapie nicht ansprechen.

Seit einigen Jahren erlauben molekularbiologische Techniken, wie die eubakterielle PCR, in einem gewissen Prozentsatz der Fälle einen Erreger trotz negativer Kulturen zu identifizieren, vor allem bei antibiotischer Vorbehandlung. Aussagen über das antibiotische Resistenzprofil des Erregers lassen diese Methoden aber derzeit noch nicht $\mathrm{zu}$.

Ein besonderes diagnostisches Problem stellt die tuberkulöse Osteomyelitis der Wirbelsäule dar (im englischen Sprachgebrauch auch „Pott's disease“ genannt), da die mikroskopische Diagnostik (säurefeste Färbung) und Histologie eine geringe Sensitivität aufweisen. Auch hier gibt es Fortschritte im Bereich der molekularen Diagnostik, die Kultur bleibt jedoch weiterhin der Goldstandard. Das Ansetzen des Mantoux-Tests sollte grundsätzlich bei jedem Tuberkuloseverdacht erwogen werden.

\section{Therapie}

Die meisten Patienten mit infektiöser Spondylitis können konservativ mit einer
Z Rheumatol 2006 $65: 7-11$

DOI 10.1007/s00393-005-0017-0

c) Springer Medizin Verlag 2006

\section{B. Huttner · M. Opravil}

\section{Die infektiöse Spondylitis}

\section{Zusammenfassung}

Bei der infektiösen Spondylitis handelt es sich in der Regel um eine Osteomyelitis von 2 benachbarten Wirbelkörpern unter Beteiligung der Zwischenwirbelscheibe (Spondylodiszitis). Am häufigsten ist die Lendenwirbelsäule betroffen, gefolgt von der Brustwirbelsäule. Die Symptome der infektiösen Spondylitis sind unspezifisch, was nicht selten zu einer Verzögerung von einigen Wochen bis zur Diagnosestellung führt. Die infektiöse Spondylitis kann durch eine Vielzahl von Erregern bedingt sein, die überwiegend auf hämatogenem Weg die Wirbelsäule erreichen. Der

\section{Infectious spondylitis}

\section{Abstract}

Infectious spondylitis usually involves osteomyelitis in two adjacent vertebral bodies and the intervertebral disc (spondylodiscitis). The most common location is the lumbar spine, followed by the thoracic spine. Symptoms are nonspecific, leading to a delay in diagnosis, in many cases, of several weeks. A large number of infectious agents can cause vertebral osteomyelitis, usually reaching the vertebra by hematogenous spread. The most commonly isolated agent häufigste Erreger ist Staphyloccocus aureus. Die Spondylitis ist zudem die häufigste skelettale Manifestation der Tuberkulose. Wie bei anderen Formen der Osteomyelitis ist die Kenntnis des Erregers für die Wahl einer adäquaten Therapie entscheidend, weshalb eine mikrobiologische Diagnose auf jeden Fall angestrebt werden sollte. Die Mehrheit der Fälle kann konservativ mit antibiotischer Therapie geheilt werden.

\section{Schlüsselwörter} Infektiöse Spondylitis · Vertebrale Osteomyelitis · Spondylodiszitis

is Staphylococcus aureus. Spondylitis remains the most common skeletal manifestation of tuberculosis. As with other forms of osteomyelitis, microbiological diagnosis is essential for the choice of adequate therapy. The majority of cases can be cured with antibiotic therapy alone.

\section{Keywords}

Infectious spondylitis · Vertebral osteomyelitis · Spondylodiscitis 


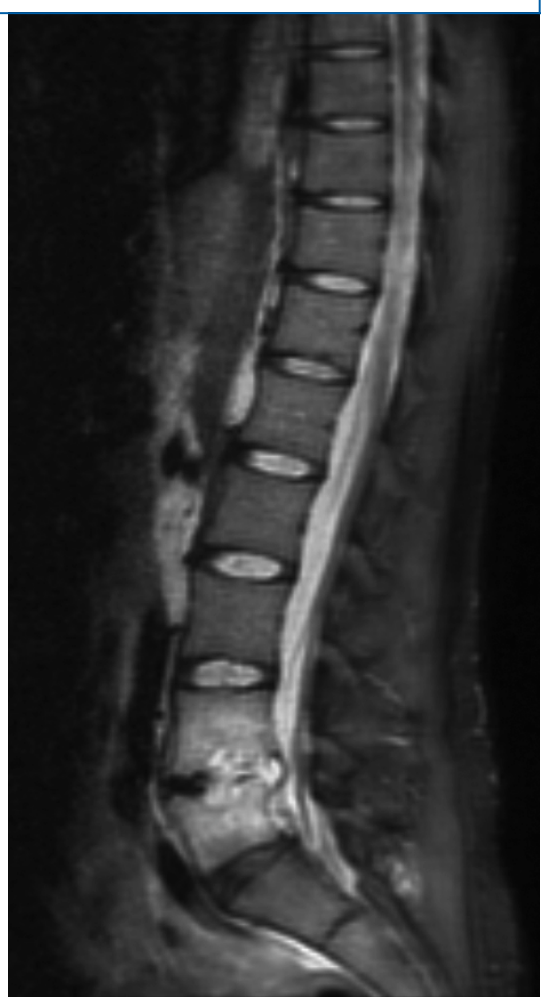

Abb. 1 \ 30-jährige HIV-positive Patientin mit intravenösem Drogenabusus, die sich mit seit 4 Wochen dauernden Rückenschmerzen und intermittierendem Fieber vorstellte. Das MRT zeigte auf Höhe von L4/ L5 eine stark destruierte Bandscheibe mit vermehrter Signalalteration der angrenzenden Wirbelkörper und ausgeprägtem Enhancement nach Gadolinium-Applikation, kompatibel mit einer vertebralen Osteomyelitis. In der Blutkultur Wachstum von Pseudomonas aeruginosa

antibiotischen Therapie geheilt werden. Aufgrund der Komplexität dieses Krankheitsbildes empfiehlt es sich meist, einen Infektiologen hinzuzuziehen. Wie bei anderen Lokalisationen der Osteomyelitis sollte eine mikrobiologische Diagnose auf jeden Fall angestrebt werden, da nur so eine resistenzgerechte, an den Erreger angepasste antibiotische Therapie möglich ist. Dies ist um so wichtiger, da sich die Behandlung über Wochen bis Monate hinzieht und die daraus resultierenden Kosten und Nebenwirkungen nicht zu vernachlässigen sind.

Mit dem Beginn einer empirischen antibiotischen Therapie sollte bis nach Gewinn von Material zur mikrobiologischen und histopathologischen Untersuchung abgewartet werden. Angesichts der in Zürich niedrigen Prävalenz von Methicillinresistentem Staphylococcus aureus (MR-
SA) bevorzugen wir einen empirischen Therapiebeginn mit einem Penicillinasefesten Penicillin (z. B. Flucloxacillin, intravenös) und einem Chinolon (z. B. Ciprofloxacin, oral), wodurch die häufigsten Erreger abgedeckt sind. Nach Erhalt der mikrobiologischen Resultate sollte die Therapie resistenzgerecht angepasst werden. Auf die spezifischen Therapieempfehlungen für die jeweiligen Erreger soll hier nicht eingegangen werden.

Über die optimale Behandlungsdauer der vertebralen Osteomyelitis gibt es keine klaren Daten aus prospektiven Studien. Initial ist eine parenteral antibiotische Therapie indiziert, da so ausreichend hohe Spiegel im Gewebe erreicht werden. Ausnahme sind Antibiotika mit guter oraler Bioverfügbarkeit (z. B. Chinolone, Rifampicin), die von Anfang an per os gegeben werden können. Bei positivem klinischen (Schmerzreduktion) und laborchemischem (Regredienz der Entzündungsparameter falls initial erhöht) Verlauf kann die Therapie nach 2-4 Wochen auf perorale Antibiotika umgestellt werden. In der angloamerikanischen Literatur wird meist eine länger dauernde parenterale Therapie bevorzugt.

Bei der Wahl der Antibiotika sollte nebst der oralen Bioverfügbarkeit auch die Gewebepenetration berücksichtigt werden. Chinolone und Clindamycin eignen sich hinsichtlich dieser beiden Eigenschaften besonders. Rifampicin spielt aufgrund der guten Biofilmpenetration eine wichtige Rolle bei Anwesenheit von Fremdmaterial.

Die antibiotische Behandlung sollte über mindestens 6 Wochen erfolgen. Bei immunsupprimierten Patienten, ausgedehnten Infektionen oder verzögertem klinischen Ansprechen sollte erwogen werden, die Therapie auf 3 Monate auszudehnen.

Besonders problematisch gestaltet sich die Therapie bei postoperativen Infekten mit Anwesenheit von Fremdmaterial, da Bakterien in dieser Situation einen Biofilm bilden, der die Wirksamkeit der antibiotischen Therapie auf vielfache Weise reduziert [5]. Idealerweise wird deshalb sämtliches Fremdmaterial entfernt, was jedoch bei frühen postoperativen Infekten meist nicht möglich ist, da dies die Stabilität der Wirbelsäule kompromittieren wür- de. Nach Débridement und initialer parenteraler Therapie empfehlen manche Autoren deshalb eine antibiotische Suppressionstherapie bis die Wirbelkörper radiologisch fusioniert sind und das Fremdmaterial entfernt werden kann [8].

Eine chirurgische Therapie ist heute nur noch bei Progression der Erkrankung trotz antibiotischer Therapie, bei Instabilität der Wirbelkörper oder zur Drainage von Abszessen indiziert. Eine adäquate antibiotische Therapie vorausgesetzt, besitzt die infektiöse Spondylitis eine gute Prognose. Bei Verzögerung der Diagnose und Behandlung können aber auch heute noch schwere Folgeschäden entstehen.

\section{Korrespondierender Autor Dr. B. Huttner}

Klinik für Infektionskrankheiten und Spitalhygiene, Departement für Innere Medizin, Universitätsspital Zürich, Rämistraße 100, 8091Zürich, E-Mail:Benedikt.Huttner@gmx.de

Interessenkonflikt: Es besteht kein Interessenkonflikt. Der korrespondierende Autor versichert, dass keine Verbindungen mit einer Firma, deren Produkt in dem Artikel genannt ist, oder einer Firma, die ein Konkurrenzprodukt vertreibt, bestehen. Die Präsentation des Themas ist unabhängig und die Darstellung der Inhalte produktneutral.

\section{Literatur}

1. Almeida A (2005) Tuberculosis of the spine and spinal cord. Eur J Radiol 55:193-201

2. Beronius M, Bergman B, Andersson R (2001) Vertebral osteomyelitis in Goteborg, Sweden: a retrospective study of patients during 1990-95 Scand J Infect Dis 33:527-532

3. Cahill D, Love L, Rechtine G (1991) Pyogenic osteomyelitis of the spine in the elderly. J Neurosurg 74:878-886

4. Chelsom J, Solberg CO (1998) Vertebral osteomyelitis at a Norwegian university hospital 1987-1997: clinical features, laboratory findings and outcome. Scand J Infect Dis 30:147-151

5. Costerton JW, Stewart PS, Greenberg EP (1999) Bacterial biofilms: a common cause of persistent infections. Science 284:1318-1322

6. Hadjipavlou AG, Mader JT, Necessary JT, Muffoletto AJ (2000) Hematogenous pyogenic spinal infections and their surgical management. Spine 25:1668-1679

7. Lew D, Waldvogel A (2004) Osteomyelits. Lancet 364:369-379

8. Mandell G, Bennett J, Dolin R (2005) Principles and p ractice of infectious diseases. Elsevier Churchill Livingstone, Philadelphia

9. McHenry M, Easley K, Locker G (2002). Vertebral osteomyelitis: long-term outcome for 253 patients from 7 Cleveland-area hospitals. Clin Infect Dis 34:1342-1350 
10. Nolla JM, Ariza J, Gomez-Vaquero C, Fiter J et al. (2002). Spontaneous pyogenic vertebral osteomyelitis in nondrug users. Semin Arthritis Rheum 31:271-278

11. Repanti M, Korovessis PG, Stamatakis MV et al. (1998) Evolution of disc degeneration in lumbar spine: a comparative histological study between herniated and postmortem retrieved disc specimens. J Spinal Disord 11:41-45

12. Schnöring M, Brock M (2003) Antibiotikaprophylaxe bei lumbalen Bandscheibenoperationen: Eine Analyse von 1030 Operationen. Zentralbl Neurochir 64:24-29

13. Solis-Garcia del Pozo J, Martinez-Alfaro E, Abad L, Solera J (2000) Vertebral osteomyelitis caused by Streptococcus agalactiae. J Infect 41:84-90

14. Stabler M, Reiser MF (2001) Imaging of spinal infection. Radiol Clin North Am 39:115-135

15. StumpeK, ZanettiM, WeishauptD et al. (2002) Positron emission tomography for differentiation of degenerative and infectious endplate abnormalities in the lumbar spine detected on MR imaging. AJR Am J Roentgenol 179:1151-1157

16. Torda AJ, Gottlieb T, Bradbury R (1995) Pyogenic vertebral osteomyelitis: analysis of 20 cases and review. Clin Infect Dis 20:320-328

\section{Rosenow, V. Tronnier, H. Göbel Neurogener Schmerz}

Management von Diagnostik und Therapie

Heidelberg: Springer Verlag GmbH 2005, 379

S., 158 Abb., 56 Tab., (ISBN 3-540-21482-8), mit CD-ROM, 79.95 EUR

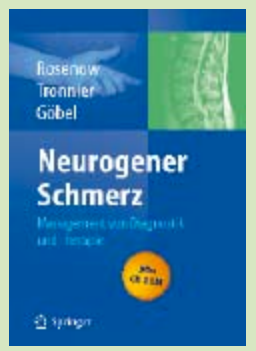

Dieses Buch mit 365

Textseiten ist ein

wirklich interdis-

ziplinäres Werk von

29 Autoren unter-

schiedlicher Prove-

nienz. Sie kommen

ebenso aus der Ana-

tomie, Physiologie

und klinischen Neuropharmakologie wie aus der Neurologie, Neurochirurgie, Neuropsychologie, Anästhesie und selbst der Industrie. Zunächst werden Anatomie und Physiologie der neuropathischen Schmerzen sowie pharmakologische Grundlagen behandelt. Es folgen Klassifikation und Terminologie des Schmerzes, die Schmerzmessung mit ihren unterschiedlichen Methoden, bevor es dann zur klinischen und bildgebenden Untersuchung und schließlich zur Behandlung übergeht. Alle Kapitel sind didaktisch sehr klar. Dies gilt auch für die Abbildungen und die Tabellen. Wichtige Feststellungen und Tipps für die Praxis sind optisch besonders hervorgehoben. Beispielhaft seien die medikamentösen Empfehlungen genannt. Einzigartig ist auch ein immerhin 38 Seiten langes Kapitel über die Bildgebung mit vielen klar beschrifteten Abbildungen hoher Druckqualität.

Der breite Raum, welcher der Bildgebung beigemessen wird, steht nicht in Widerspruch zum Prinzip des Primats der Schmerzanamnese und klinischen Untersuchung. In dem sehr kompakten Kapitel über die neurologische Untersuchung hat mir die Aufforderung gefallen, bei der Erhebung der Eigenanamnese auch auf vorangegangene Operationen zu achten und deren genaue medizinische Begründung zu erforschen, weil sich hinter solchen Operationen nämlich „häufig Versuche einer frustranen Schmerztherapie verbergen".

Dem Buch ist eine CD beigefügt. Sie enthält zwei Videofilme mit einem vor- geschalteten kurzen einführenden Text. Der eine Film hat die Implantation einer Schmerzpumpe, der andere die Implantation einer SCS-Elektrode zur Teststimulation zum Inhalt.

Bei diesem Buch handelt es sich um ein ebenso wissenschaftliches wie pragmatisches Werk, welches alle Aspekte der Diagnostik und Therapie vor allem chronischer Schmerzsyndrome behandelt. Für die medikamentöse Therapie finden sich sehr konkrete Dosierungsrichtlinien und Diskussionen von Alternativen. Und auch die operativen Behandlungsmöglichkeiten werden von wirklichen Fachleuten differenziert dargestellt.

Es ist durchgehend zu erkennen, dass alle Autoren auf ihrem Gebiet nicht nur hohe theoretische, sondern auch praktische Erfahrungen besitzen.

Ich wünsche dem Buch, dass es jeder Arzt zur Hand nehmen und nutzen möge, der mit Schmerzpatienten zu tun hat.

Hans-Peter Richter (UIm) 